\title{
Molecular Evolution of the Puroindoline-a, Puroindoline-b, and Grain Softness Protein-1 Genes in the Tribe Triticeae
}

\author{
Alicia N. Massa, ${ }^{1}$ Craig F. Morris ${ }^{2}$ \\ ${ }^{1}$ Department of Crop \& Soil Sciences, Washington State University, Pullman Washington 99164-6394, USA (affiliated with the USDA \\ ARS Western Wheat Quality Laboratory) \\ ${ }^{2}$ USDA ARS Western Wheat Quality Laboratory, E-202 Food Science \& Human Nutrition Facility East, WSU, Pullman Washington \\ 99164-6394, USA
}

RE: D0I: 10.1007/s00239-005-0292-z. J Mol Evolution 63(4):526-536. Table 2, the line for Gsp-1, D (T. aestivum) - the reverse primer 5'-AGTGATGG
GATGTTGCAGA-3' appeared incorrectly (missing a ' $G$ '). The correct sequence is 5'-AGTGATGGG GATGTTGCAGA-3’. 